05

\title{
Нелинейные упругие волны в твердотельном изотропном клине с дефектами
}

\author{
() А.И. Коробов, А.А. Агафонов, М.Ю. Изосимова
}

Московский государственный университет им. М.В. Ломоносова, 119991 Москва, Россия

e-mail: aikor42@mail.ru

(Поступило в Редакцию 12 июля 2017 г.)

\begin{abstract}
Приведены результаты экспериментальных исследований линейных и нелинейных свойства клиновых упругих волн в образцах остроугольных клиньев из изотропного поликристаллического сплава Д16 с дефектами. Методом лазерной виброметрии исследована локализация волн у ребра клина. В интервале частот $(0.25-1.5 \mathrm{MHz})$ импульсным методом измерены скорости клиновых волн. Дисперсии при этом не выявлено. Обнаружена генерация второй гармоники клиновых волн. Исследованы зависимости скорости и амплитуды второй гармоники от амплитуды первой гармоники клиновых волн. Отмечено, что наблюдаемые нелинейные эффекты в клиновых волнах не могут быть объяснены в рамках классической пятиконстантной теории упругости Мурнагана. Эти эффекты объясняются наличием в исследуемых образцах структурной нелинейности, обусловленной дефектной структурой в материале клина.
\end{abstract}

DOI: 10.21883/JTF.2018.03.45595.2422

\section{Введение}

Клиновые упругие волны (КУВ) впервые были обнаружены численными расчетами в 1972 г. независимо Лагассом [1] и Марадудиным с сотрудниками [2]. Согласно численным расчетам [3-5], вдоль ребра твердотельных клиньев с углами раскрыва от 0 до $90^{\circ}$ могут распространяться несколько антисимметричных (изгибных) локализованных мод и одна симметричная мода КУВ. В настоящей работе будут рассматриваться только изгибные КУВ. Экспериментальные исследования показали, что энергия этих мод сконцентрирована в окрестности вершины клина, смещение частиц в изгибной КУВ в основном происходит в направлении перпендикулярном плоскости, разделяющей пополам угол при вершине клина, и экспоненциально затухает с расстоянием от ребра клина. Скорости изгибных КУВ существенно ниже скорости рэлеевской волны на плоской боковой поверхности клина. В [1] была предложена эмпирическая формула для скоростей мод КУВ в остроугольном клине $V_{n}$ :

$$
V_{n}=V_{R} \sin (n \theta) \quad \text { при } n \theta<90^{\circ},
$$

где $V_{R}$ - скорость волны Рэлея на плоской боковой поверхности клина, $n-$ номер антисимметричной моды КУВ, $\theta-$ угол раскрыва клина.

Впервые теоретическое рассмотрение нелинейных эффектов в антисимметричных КУВ в остроугольном изотропном клине проведено в [6]. Из [6] следует, что в изотропных твердых телах ввиду их высокой симметрии генерация второй упругой гармоники изгибных КУВ невозможна. Однако в твердых телах с дефектами (дислокациями, микротрещинами, нарушениями сплошности, остаточными механическими напряжениями) наряду c нелинейностью сил межмолекулярного взаимодействия (классическая нелинейность) проявляется струк- турная (неклассическая) упругая нелинейность, приводящая к нелинейной связи между механическим напряжением и деформацией. Структурная нелинейность может на несколько порядков превышать классическую упругую нелинейность [7]. Возможные механизмы структурной нелинейности рассмотрены в [8].

Неклассическая нелинейность приводит к новым нелинейным упругим явлениям в изотропных твердых телах с дефектами: генерации второй сдвиговой и изгибной упругих гармоник, эффектам быстрой и медленной динамики [9-15]. Указанные нелинейные явления не наблюдаются в бездефектных изотропных твердых телах. Нелинейные упругие явления, проявляемые при распространении КУВ в изотропном упругом клине, могут служить диагностическими признаками наличия в материале клина дефектов.

В [16] отмечается, что КУВ могут быть использованы в неразрушающем контроле для диагностики композитных материалов лопаток турбин, режущих кромок инструментов, различных рельсовых конструкций. Однако использование КУВ в области неразрушающего контроля твердых тел в настоящее время тормозится отсутствием информации о влиянии различных дефектов в материале клина: микротрещин, расслоений, остаточных упругих напряжений, а также влияние постоянных или переменных нагрузок на нелинейные упругие свойства волн в твердотельном клине.

Целью настоящей работы являются экспериментальные исследования влияния дефектов и механической силы, приложенной параллельно ребру клина на линейные и нелинейные упругие свойства КУВ в образцах из поликристаллического сплава алюминия Д16. Сплав Д16 широко применяется в машиностроении, автомобилестроении и в авиастроении. 


\section{1. Экспериментальные образцы и методика эксперимента}

\section{1. Образцы}

Для проведения экспериментальных исследований упругих свойств КУВ из цилиндрических прутков диаметром 40 и $12 \mathrm{~mm}$ изотропного поликристаллического сплава алюминия Д16 были изготовлены образцы клиньев. В заготовках предварительно были образованы остаточные деформации. Образец № 1, изготовленный из прутка диаметром $40 \mathrm{~mm}$ и длиной $180 \mathrm{~mm}$, имел форму прямоугольной призмы, основаниями которой служат прямоугольные треугольники с углами $30^{\circ}$ и $60^{\circ}$ (рис. $1, a$ ). В этом образце наклепом были созданы остаточные деформации. Образец № 2 (рис. 1,b) был изготовлен из цилиндрического прутка диаметром $12 \mathrm{~mm}$ из сплава Д16 с остаточными сдвиговыми деформациями, которые создавались при вращении прутка, закрепленного на одном конце, вокруг его оси в токарном станке. После снятия крутящего момента в прутке оставались деформации сдвига, микротрещины, дислокации. Величина остаточных сдвиговых деформаций рассчитывалась по величине изгиба образующей на поверхности цилиндрического стержня и была равна 0.3 . Изготовленный клин имел длину $10 \mathrm{~cm}$ и апертуру угла $44^{\circ}$. Для закрепления образца в растягивающем устройстве в его торцах были просверлены отверстия диаметром 6 mm, в которых была нарезана резьба.
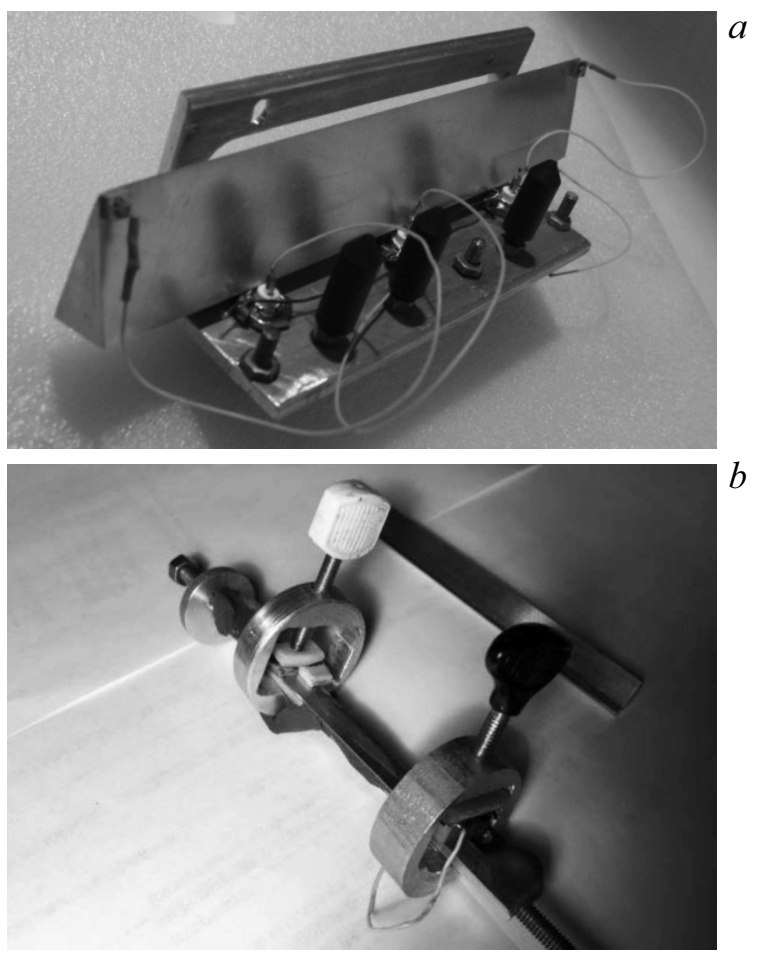

Рис. 1. Экспериментальные образцы: образец № $1(a)$, образец № $2(b)$.

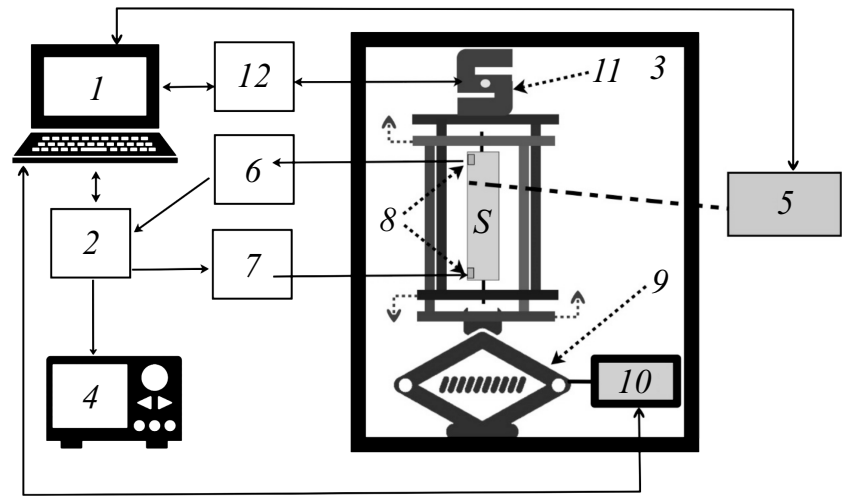

Рис. 2. Блок-схема автоматизированной экспериментальной установки для исследования линейных и нелинейных упругих свойств КУВ: $S-$ образец, 1 - персональный компьютер, 2 ультразвуковой автоматизированный комплекс Ritec RAM5000 SNAP SYSTEM, 3 - система для создания контролируемого одноосного растяжения, 4 - цифровой осциллограф DS09104A, 5 - допплеровский виброметр PSV-300, 6, 7 фильтры низких и высоких частот, 8 - пьезоэлектрические преобразователи, $9-$ винтовой домкрат, $10-$ шаговый двигатель, 11 - датчик силы, 12 - АЦП.

\section{2. Экспериментальные методы}

Экспериментальные исследования упругих свойств КУВ в твердотельных клиньях проводились на автоматизированной ультразвуковой установке, блок-схема которой приведена на рис. 2. Она состоит из персонального компьютера (ПК) 1, ультразвукового автоматизированного комплекса Ritec RAM-5000 SNAP SYSTEM 2 и системы 3 для создания контролируемого одноосного растяжения исследуемого образца $S$. Для наблюдения за упругими импульсами в клине использовался цифровой осциллограф DS09104A (Agilent, США) 4. С помощью лазерного сканирующего допплеровского виброметра PSV-300 (Polytec, Германия) 5 выполнялась визуализация колебаний плоской поверхности одной из боковых граней образца вблизи ребра клина. Комплекс 2 работает в импульсном режиме. Он позволяет исследовать линейные и нелинейные упругие свойства твердых тел. Для исследования нелинейных упругих свойств твердых тел в комплексе реализован динамический спектральный метод, заключающийся в исследовании эффективности генерации второй упругой гармоники на частоте $2 f$ в исследуемом образце при распространении в нем упругой волны конечной амплитуды на частоте $f$. Комплекс позволяет измерять зависимость амплитуды первой и второй упругих гармоник от амплитуды зондирующего сигнала в автоматическом режиме. Результаты измерений оцифровывались встроенными в измерительный комплекс 16-разрядными аналого-цифровыми преобразователями (АЦП) и передавались в ПК. Для выделения сигналов первой и второй гармоник КУВ были разработаны фильтры низких и высоких частот 6,7. Возбуждение и прием КУВ в образцах проводилось 

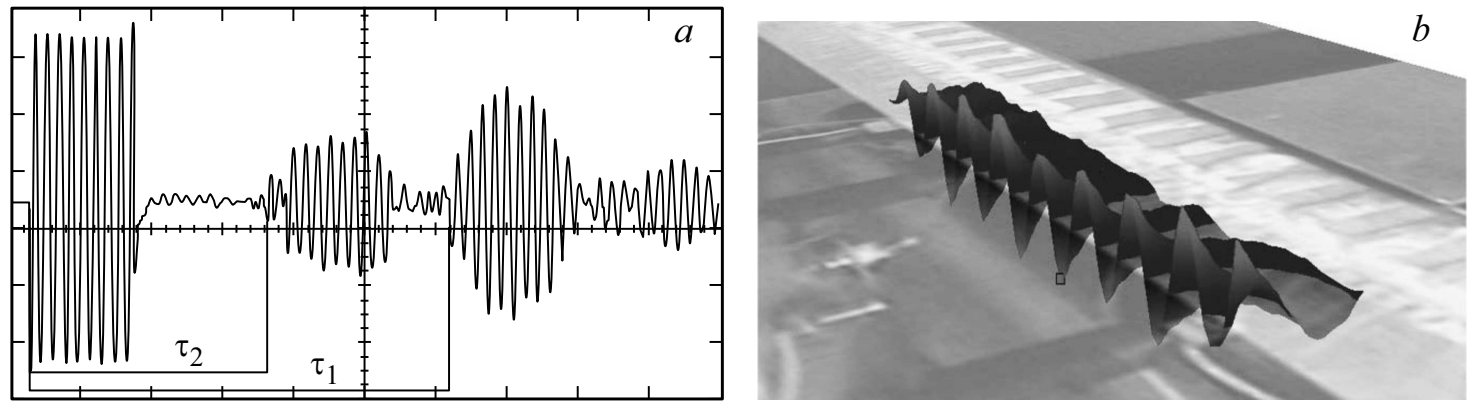

Рис. 3. Серия импульсов двух первых мод КУВ в $30^{\circ}$ клине на экране осциллографа (a); результаты визуализации КУВ в $60^{\circ}$ клине $(b)$.

пьезоэлектрическими преобразователями 8 , прикрепленными на боковые поверхности клина непосредственно у его ребра.

Контролируемое одноосное растяжение образца в системе 3 обеспечивалось винтовым домкратом 9 с приводом от шагового двигателя 10, который управлялся ПК. Это позволяло изменять приложенную к образцу силу по заданной программе. Величина силы измерялась электронным датчиком 11 , сигнал с которого оцифровывался 16-разрядным АЦП 12 (National Instruments, США). Цифровая информация с ультразвукового комплекса 2 и АЦП 12 в ходе эксперимента поступала в ПК 1 для ее хранения и последующего анализа. Для управления работой комплекса и проведения ультразвуковых измерений с автоматической регистрацией результатов ультразвукового эксперимента и их анализа для ПК был разработан пакет программ.

\section{2. Экспериментальные результаты и их обсуждение}

\section{1. Линейные свойства клиновых волн}

Излучаемый и принимаемый сигналы с комплекса 2, соответствующие КУВ в исследуемом образце, поступали в ПК и регистрировались на экране цифрового осциллографа (рис. 3,a). В принимаемом сигнале наблюдалось несколько импульсов как КУВ различных мод, так и волн Лэмба, поверхностных волн, а также различных отражений от торцов клина перечисленных волн. (Особенности поведения упругих волн у ребра клина рассмотрены в [17].) Для уменьшения паразитных импульсов в принимаемом сигнале на торцы клина (для минимизации отражений) и на части клина, удаленные от его ребра на расстоянии, превышающим длину волны КУВ $\lambda$ в несколько раз, наносился поглотитель. В качестве поглощающего материала использовался пластилин.

При возбуждении КУВ в $30^{\circ}$ клине на осциллографе регистрировалось два импульса (рис. $3, a$ ), соответствующих первой и второй модам КУВ со скоростями, равными $1490 \pm 60$ и $2590 \pm 120 \mathrm{~m} / \mathrm{s}$ соответственно. Ско- рость КУВ определялась стандартным эхо-импульсным методом путем измерения времени распространения импульсов КУВ в клине $\tau_{1}$ и $\tau_{2}$. В $60^{\circ}$ клине на осциллографе наблюдался один импульс, соответствующий единственной моде КУВ в этом клине, скорость которой была равна $2580 \pm 120 \mathrm{~m} / \mathrm{s}$. В клине с апертурой угла $44^{\circ}$ наблюдался один импульс КУВ со скоростью $1820 \pm 100 \mathrm{~m} / \mathrm{s}$. Скорость волны Рэлея на плоской боковой поверхности клиньев вдали от его ребра была равна $V_{R}=2900 \pm 130 \mathrm{~m} / \mathrm{s}$. Значения величин скоростей упругих волн в клиньях согласуются с эмпирической формулой (1).

В $60^{\circ}$ клине в интервале частот $0.25-1.5 \mathrm{MHz}$ были проведены измерения скорости КУВ волн в зависимости от частоты. Дисперсии скорости КУВ в указанном диапазоне частот обнаружено не было. Это находится в согласии с численными расчетами [1-5].

С помощью лазерного сканирующего допплеровского виброметра PSV-300 была выполнена визуализация амплитуды колебаний ребра $60^{\circ}$ клина и прилегающей к нему плоской боковой грани на частоте $250 \mathrm{kHz}$, вызванные КУВ (рис. 3, $b$ ).

Измерение амплитуды колебаний в КУВ перпендикулярно ребру клина с помощью лазерного виброметра позволило определить зависимость амплитуды колебаний $A$ КУВ от расстояния от ребра клина $Y$. Экспериментальная зависимость амплитуды КУВ $A$ от расстояния $Y$, нормированного на длину волны КУВ $\lambda$, аппроксимируется экспонентой

$$
A=4 \cdot 10^{-8} \exp (2.5 Y / \lambda)
$$

и согласуется с результатами численных расчетов, выполненных в [3-5].

\section{2. Нелинейные упругие свойства КУВ}

Исследование упругих нелинейных свойств КУВ в работе проводилось следующими методами.

1. Экспериментально исследован эффект быстрой динамики: зависимость скорости КУВ в клине от ее амплитуды. 

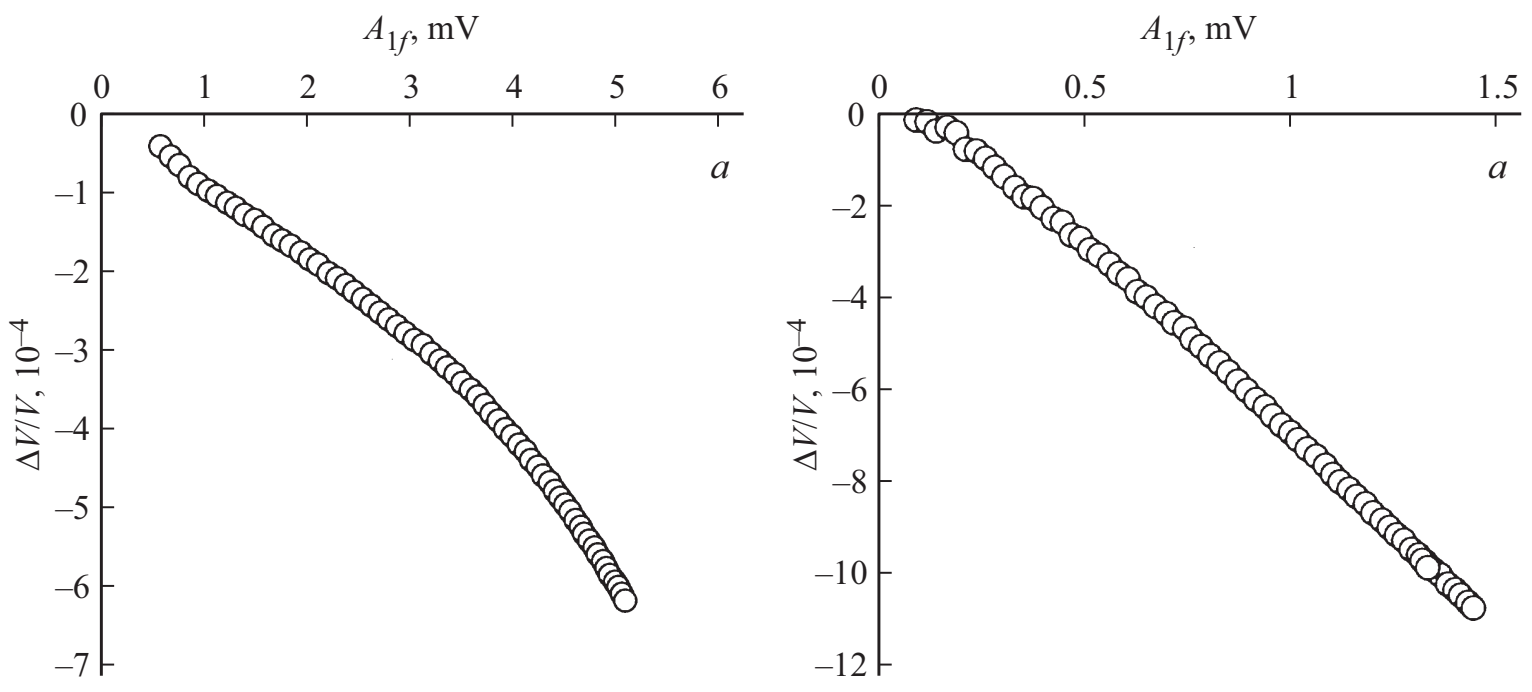

Рис. 4. Эффект быстрой динамики КУВ в клиньях с апертурой $30^{\circ}(a)$ и апертурой $60^{\circ}(b)$.

2. Спектральным методом оценивалась эффективность генерации второй гармоники КУВ.

3. Исследовано влияние статической механической силы, приложенной параллельно ребру клина, на амплитуду второй гармоники КУВ в клине.

Экспериментальные исследования зависимости относительного изменения скорости КУВ $\Delta V / V$ от ее амплитуды (эффект быстрой динамики) проводились в образце № 1. Для экспериментальных исследований были выбраны: вторая мода КУВ в 30 клине образца № 1 на частоте $494 \mathrm{kHz}$ и единственная мода КУВ в $60^{\circ}$ клине образца № 1 на частоте $399 \mathrm{kHz}$. Скорости КУВ этих мод в пределах ошибки эксперимента совпадали. Результаты исследований эффекта быстрой динамики КУВ приведены на рис. 4. Как видно из рисунка, эффект быстрой динамики КУВ в $60^{\circ}$ клине проявляется сильнее по сравнению с аналогичным эффектом в $30^{\circ}$ клине. Это связано с особенностями локализации основной моды КУВ в $60^{\circ}$ клине и второй клиновой моды в $30^{\circ}$ клине. Обнаруженный эффект быстрой динамики КУВ указывает на наличие в нем дефектов, приводящих к возникновению в клине структурной нелинейности.

Генерация второй гармоники КУВ экспериментально исследована в $30^{\circ}$ и $60^{\circ}$ клиньях (образец № 1) и в $44^{\circ}$ клине (образец №2). В спектре гармонической КУВ конечной амплитуды, прошедшей через образец, во всех исследованных в работе образцах клиньев, помимо КУВ на частоте зондирующей волны $f$ с амплитудой $A_{1 f}$, также зарегистрирована вторая гармоника КУВ с амплитудой $A_{2 f}$ на частоте $2 f:$ в $30^{\circ}$ клине $(f=494 \mathrm{kHz}, 2 f=988 \mathrm{kHz})$, в $60^{\circ}$ клине $(f=399 \mathrm{kHz}, 2 f=998 \mathrm{kHz})$ и в $44^{\circ}$ клине $(f=300 \mathrm{kHz}, 2 f=600 \mathrm{kHz})$. Результаты экспериментальных исследований зависимости амплитуды второй гармоники КУВ от амплитуды первой гармоники КУВ в $30^{\circ}, 60^{\circ}$ и $44^{\circ}$ клиньях приведены на рис. 5, a, c,e.
Спектральный состав прошедшей через образец клиновой волны конечной амплитуды описывается следующим выражением:

$$
A=A_{1 f} \sin \left(2 \pi f t-k_{f} L\right)+A_{2 f} \sin \left[2\left(2 \pi f t-k_{f} L\right)\right],
$$

где $k_{f}$ - волновой вектор первой гармоники волны, $L$ - расстояние между излучающим и приемным преобразователями КУВ, $f-$ частота сигнала, $t-$ время. Зависимость амплитуды второй гармоники $A_{2 f}$ от амплитуды основной волны $A_{1 f}$ в твердых телах определяется следующим уравнением [18]:

$$
A_{2 f}=|N| k_{f}^{2} L A_{1 f}^{M} / 8 .
$$

Параметр $N$ характеризует величину упругой нелинейности в образце, а параметр $M-$ механизм упругой нелинейности. Необходимо отметить, что амплитуды первой и второй гармоник КУВ экспериментально измерялись по абсолютной величине. Фазы этих величин не измерялись. Поэтому в (2) нелинейный акустический параметр $N$ записан по абсолютной величине.

В бездефектном твердом теле упругая нелинейность определяется ангармонизмом межатомного взаимодействия. В этом случае в выражении (2) показатель степени $M=2$. Однако этот механизм упругой нелинейности не приводит к генерации второй гармоники изгибной КУВ в изотропных твердых телах [6], но в средах со структурной нелинейностью, которая обусловлена дефектами, генерация второй гармоники КУВ возможна [9]. В этом случае показатель степени $M=1[9,19]$. На рис. 5, $a, c, e$ видно, что во всех исследованных в работе клиньях амплитуда второй гармоники $A_{2 f}$ КУВ практически линейно зависит от амплитуды первой гармоники $A_{1 f}$ КУВ. Для уточнения этого предположения экспериментальные результаты зависимости амплитуды 

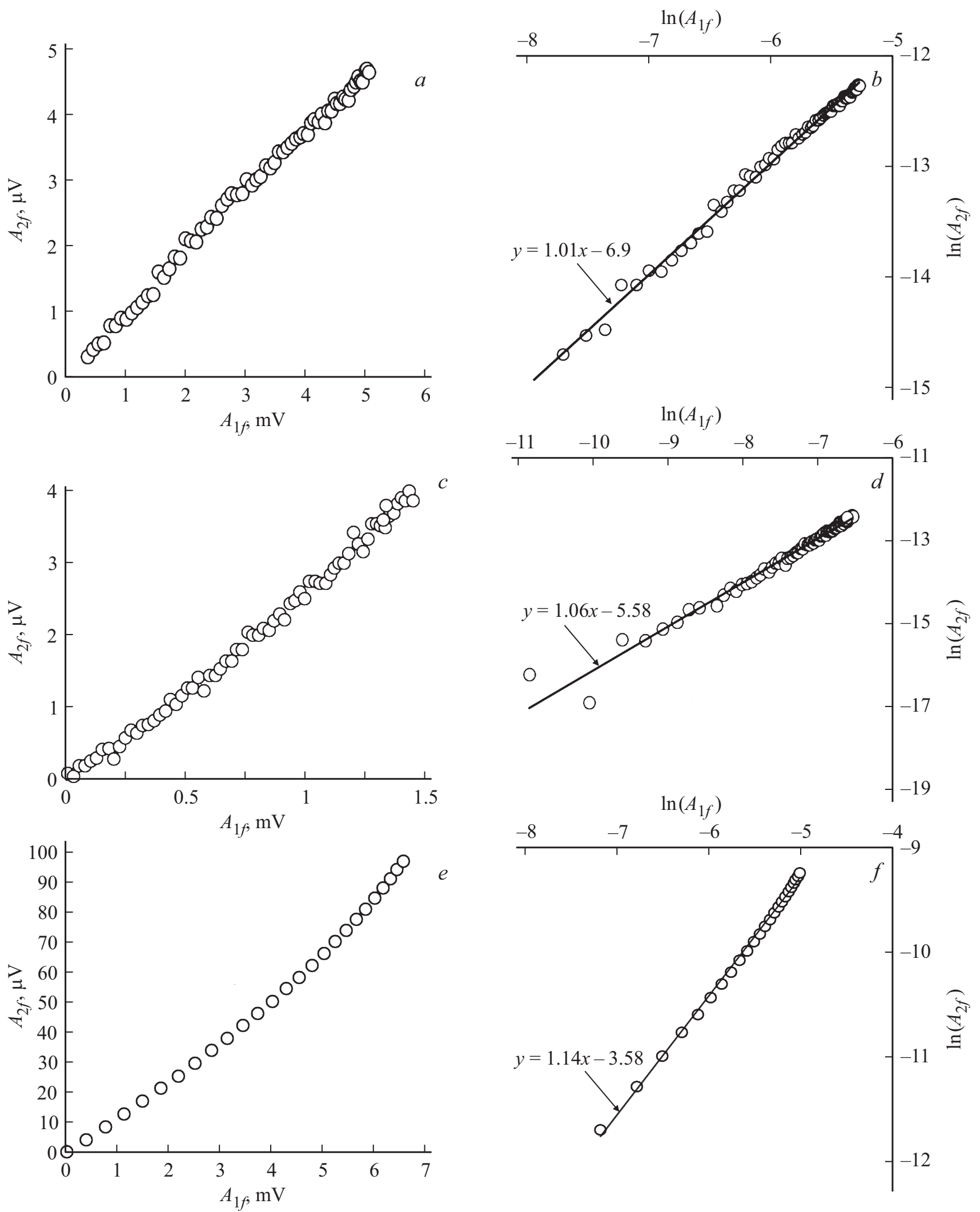

Рис. 5. Зависимость амплитуды второй гармоники клиновой волны $A_{2 f}$ от амплитуды первой гармоники $A_{f}$ в линейном масштабе: $(a)$ в $30^{\circ}$ клине, $(c)$ в $60^{\circ}$ клине, $(e)$ в $44^{\circ}$ клине и в двойном логарифмическом масштабе: $(b)$ в $30^{\circ}$ клине, $(d)$ в $60^{\circ}$ клине, (f) в $44^{\circ}$ клине. 


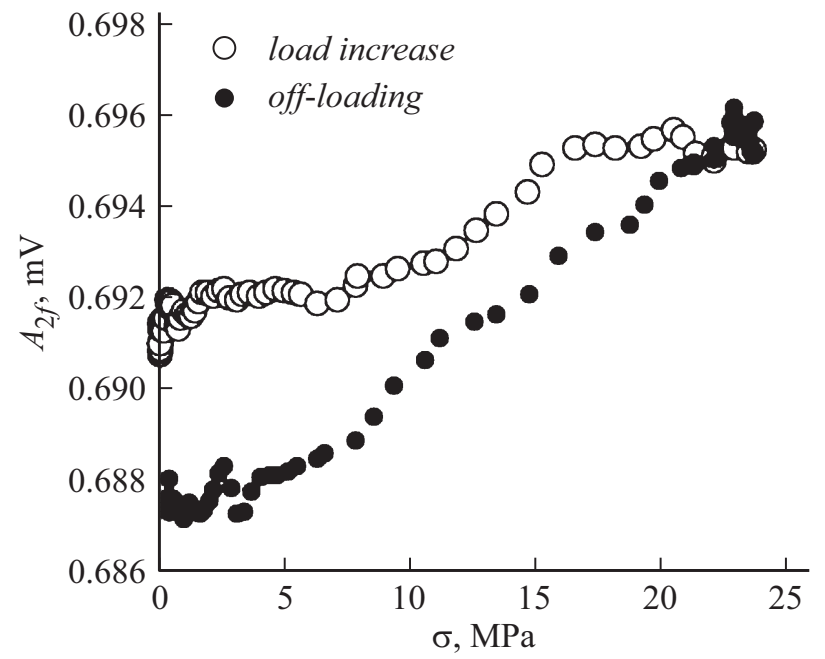

Рис. 6. Влияние механического напряжения $\sigma$ на величину амплитуды второй гармоники КУВ в клине с апертурой угла $44^{\circ}$.

второй гармоники $A_{2 f}$ от амплитуды первой $A_{1 f}$ гармоники КУВ были представлены в двойном логарифмическом масштабе. Эти зависимости с достаточной степенью точности аппроксимируются прямой $y=C x+B$ (рис. 5, $b, d, f$ ).

Для анализа результатов, приведенных на рис. 5. $b, d, f$, прологарифмируем (2):

$$
\ln A_{2 f}=\ln \left(N k_{f}^{2} L / 8\right)+M \ln A_{1 f} .
$$

Из сравнения равенства (3) с уравнением прямой $y=C x+B$ следует

$$
y=\ln A_{2 f}, \quad x=\ln A_{1 f}, \quad C=M, \quad B=\ln \left(N k_{f}^{2} L / 8\right) .
$$

Согласно (4), тангенс угла наклона аппроксимирующей прямой к оси абсцисс равен показателю степени $M$ в (2). Проведенный анализ зависимостей, приведенных на рис. $5, b, d, f$, позволил определить численные значения показателя $M$ в уравнении (2). Этот показатель оказался равным: для $30^{\circ}$ клина $M=1.01$, для $60^{\circ}$ клина $M=1.06$ и для $44^{\circ}$ клина $M=1.14$. Согласно уравнению (2) с измеренными величинами $M$, амплитуда второй гармоники $A_{2 f}$ практически линейно зависит от амплитуды первой гармоники $A_{1 f}$.

Обнаруженный эффект быстрой динамики в $30^{\circ}$ и $60^{\circ}$ клиньях (рис. 4) и практически линейная зависимость амплитуды второй гармоники $A_{2 f}$ от амплитуды первой гармоники $A_{1 f}$ в исследованных клиньях (рис. $5, b, d, f)$ не могут быть объяснены в рамках классической пятиконстантной теории упругости Мурнагана $[20]$. В $[9,19]$ показано, что линейная зависимость второй упругой гармоники $A_{2 f}$ от амплитуды первой гармоники $A_{1 f}$ наблюдается в дефектных средах. Это позволяет сделать вывод, что генерация второй гармоники КУВ и эффект быстрой динамики в изотропных образцах клиньев объясняется наличием в них дефектов, приводящих к появлению структурной нелинейности в материале клина. Обнаруженные нелинейные упругие эффекты в КУВ могут служить диагностическими признаками наличия дефектов в материале клиньев.

В клине с апертурой угла $44^{\circ}$ с искусственно созданными остаточными сдвиговыми деформациями было исследовано влияние реверсивно изменяющегося растягивающего напряжения $\sigma$ в интервале $0-25-0 \mathrm{MPa}$, приложенного параллельно ребру клина на величину амплитуды второй гармоники КУВ в нем (рис. 6). Измерения проводились в импульсном режиме на частоте зондирующего сигнала $f=300 \mathrm{kHz}$. С увеличением величины растягивающего напряжения $\sigma$ от 0 до $25 \mathrm{MPa}$ амплитуда второй гармоники практически линейно увеличивалась на $\approx 0.7 \%$, при уменьшении приложенного давления от $25 \mathrm{MPa}$ до 0 амплитуда второй гармоники КУВ уменьшалась также практически линейно на $\approx 1.4 \%$. При этом амплитуда второй гармоники при $\sigma=0$ не возвращалась к первоначальному значению. Максимальное значение приложенного к образцу статического механического напряжения $\sigma=25 \mathrm{MPa}$ значительно меньше условного предела текучести $\sigma_{0.2}=340 \mathrm{MPa} \mathrm{в}$ материале клина сплава Д16 [21]. Поэтому в интервале механических напряжений 0-25-0 MPa, приложенных к клину, материал клина находился в области упругих деформаций. Приведенная на рис. 6 зависимость амплитуды второй гармоники КУВ от реверсивно изменяющегося механического напряжения $\sigma$ вызвана не пластической деформацией образца, а связана с перестройкой дефектной структуры в материале клина.

\section{Заключение}

В работе исследованы особенности распространения КУВ в клиньях с апертурой углов $30^{\circ}, 44^{\circ}$ и $66^{\circ}$, изготовленных из поликристаллического сплава алюминия Д16, в котором предварительно были созданы дефекты. Была разработана методика возбуждения и приема КУВ в исследуемых образцах. Во всех клиньях импульсным методом измерены скорости распространения КУВ и волны Рэлея на плоской боковой поверхности клина. Значения величин скоростей упругих волн в клиньях согласуются с эмпирической формулой (1), предложенной Лагассом для скоростей мод КУВ в остроугольном клине. В $60^{\circ}$ клине в диапазоне частот $0.25-1.5 \mathrm{MHz}$ проведены измерения скорости КУВ в зависимости от частоты. Дисперсии скорости КУВ в указанном диапазоне частот обнаружено не было. C помощью лазерного сканирующего допплеровского виброметра PSV-300 была визуализирована локализация колебаний у ребра $60^{\circ}$ клина. Установлено, что КУВ локализована у ребра клина в пределе длины волны КУВ. Экспериментально исследованы нелинейные упругие свойства КУВ в образцах клиньев с дефектами. В $30^{\circ}$ и $60^{\circ}$ клиньях исследован эффект быстрой динамики: 
измерена зависимость скорости КУВ от ее амплитуды. Обнаружено, что с увеличением амплитуды КУВ ее скорость уменьшается. Эффект быстрой динамики КУВ в $60^{\circ}$ клине проявляется сильнее по сравнению с аналогичным эффектом в $30^{\circ}$ клине. Это связано с особенностями локализации основной моды КУВ в $60^{\circ}$ клине и второй клиновой моды в $30^{\circ}$ клине. В клиньях из изотропного твердого тела с дефектами впервые зарегистрирована и исследована генерация второй гармоники КУВ. Проведенные измерения обнаружили практически линейную зависимость амплитуды второй гармоники от амплитуды первой гармоники КУВ, что находится в противоречии с классической теорией нелинейной упругости твердых тел. Генерация второй гармоники КУВ в клиньях объясняется наличием в них структурной нелинейности, обусловленной дефектами в материале клиньев. В $44^{\circ}$ клине с искусственно созданными остаточными сдвиговыми деформациями было исследовано влияние реверсивно изменяющего растягивающего механического напряжения $\sigma$, приложенного параллельно ребру клина на амплитуду второй гармоники КУВ в нем. Обнаружено линейное увеличение амплитуды второй гармоники КУВ. Исследованные нелинейные упругие явления не могут быть объяснены в рамках классической пятиконстантной теории упругости Мурнагана [20]: они связаны с упругой структурной нелинейностью, обусловленной наличием в материалах клиньев дефектов. Полученные в предлагаемой работе экспериментальные результаты могут быть использованы при создании новых методов неразрушающего контроля различных материалов и конструкций, основанных на принципах нелинейной акустики.

Исследования выполнены при поддержке гранта РФФИ (проект № 17-02-01123) и программы поддержки Ведущих научных школ (грант НШ-7062.2016.2.).

\section{Список литературы}

[1] Lagasse P.E., Mason I.M., Ash E.A. // IEEE Trans. Son. Ultrason. 1973. Vol. 21. P. 225-236.

[2] Maradudin A.A., Wallis R.F., Mills D.L., Ballard R.L. // Phys. Rev. B. 1972. Vol. 6. P. 1106-1111.

[3] Moss S.L., Maradudin A.A., Cunningham S.L. // Phys. Rev. B. 1973. Vol. 8. P. 2999-3008.

[4] Sharon T.M., Maradudin A.A., Cunningham S.L. // Phys. Rev. B. 1973. Vol. 8. P. 6024-6026.

[5] McKenna J., Boyd G.D., Thurston R.N. // IEEE Trans. Son. Ultrason. 1974. Vol. 21. P. 178-186.

[6] Krylov V.V., Parker D.F. // Wave Motion. 1992. Vol. 15. P. $185-200$.

[7] Руденко О.В. // УФН. 2006. Т. 176. № 1. С. 77-95.

[8] Руденко О.В. // Дефектоскопия. 1993. № 8. С. 24-32.

[9] Зарембо Л.К., Шанин А.В. // Акустический журн. 1995. Т. 41. № 4. С. 587-590.

[10] Коробов А.И., Изосимова М.Ю. // Акустический журн. 2006. T. 52. № 5. С. 683-692.
[11] Universality of Nonclassical Nonlinearity / Ed. P. Delsanto. NY.: Springer Verlag, 2006. 539 p.

[12] Коробов А.И., Одина Н.И., Мехедов Д.М. // Акустический журн. 2013. Т. 59. № 4. С. 438-444.

[13] Korobov A.I., Izossimova M.Yu, Shirgina N.V. // Phys. Proc. Elsevier. 2015. Vol. 70. P. 415-419.

[14] Коробов А.И., Изосимова М.Ю., Одина Н.И. // Акустический журн. 2015. Т. 61. С. 317-324.

[15] Guyer R.A., Johnson P.A. Nonlinear Mesoscopic Elasticity: the Complex Behaviour of Rocks, Soil, Concrete. Wiley-VCH, 2009. $410 \mathrm{p}$.

[16] Hess P., Lomonosov A.M., Mayer A.P. // Ultrasonics. 2016. Vol. 71. P. 278-287.

[17] Толипов Х.Б. // ЖТФ. 2012. Т. 82. Вып. 9. С. 139-142.

[18] Зарембо Л.К., Красильников В.А. // УФН. 1970. Т. 102. № 4. C. 549-586.

[19] Назаров В.Е., Сутин А.М. // Акустический журн. 1989. Т. 35. Вып. 4. С. 711-716.

[20] Зарембо Л.К., Красильников В.А. Введение в нелинейную акустику. М.: Наука, 1966. 309 с.

[21] Конструкционные материалы. Справочник. / Под ред. Б.Н. Арзамасова. М.: Машиностроение, 1990. 688 с. 